\title{
mTOR signaling in the neuropathophysiology of depression: current evidence
}

REVIEW

\author{
Gislaine Z Réus' \\ João Quevedol,2 \\ Ana Lúcia S Rodrigues ${ }^{3}$ \\ 'Laboratory of Neurosciences, \\ Graduate Program in Health Sciences, \\ Health Sciences Unit, University of \\ Southern Santa Catarina, Criciúma, \\ Santa Catarina, Brazil; ${ }^{2}$ Center for \\ Translational Psychiatry, Department \\ of Psychiatry and Behavioral Sciences, \\ University of Texas Medical School, \\ Houston, TX, USA; ${ }^{2}$ Laboratory \\ of Neurobiology of Depression, \\ Department of Biochemistry, \\ Center of Biological Sciences, \\ Federal University of Santa Catarina, \\ Florianópolis, Santa Catarina, Brazil
}

Correspondence: Ana Lúcia S Rodrigues Laboratory of Neurobiology of

Depression, Department of Biochemistry, Center of Biological Sciences, Campus Universitário, Federal University of Santa Catarina, Trindade, 88040-900, Florianópolis, Santa Catarina, Brazil

Tel +55 48 372I 5043

$\mathrm{Fax}+554837219672$

Email alsrodri@gmail.com
This article was published in the following Dove Press journal:

Journal of Receptor, Ligand and Channel Research

23 November 2015

Number of times this article has been viewed
Abstract: Despite significant progress in major depressive disorder (MDD) research over the past decades, the mechanisms underlying its pathophysiology and treatment remain to be established. The complexity and heterogeneity of MDD involves multiple causes, such as inflammation, genetic, and environmental factors that could be related to poor effectiveness, variability of response to antidepressant drugs, delay in clinical response, and side effects. Ketamine, an $N$-methyl-D-aspartate receptor antagonist, has been proposed as a revolutionary antidepressant that acts rapidly and is effective for treatment-resistant MDD. Ketamine stimulates mammalian target of rapamycin (mTOR), which is involved in transcription, survival, and cell proliferation. mTOR is an emerging signaling pathway of interest in MDD pathophysiology and treatment. Thus, this review describes the role of mTOR in the pathophysiology of MDD as well as highlights therapeutic targets that modulate mTOR signaling.

Keywords: mTOR, antidepressant, major depressive disorder

\section{Introduction}

Major depressive disorder (MDD) is a highly prevalent and burdensome psychiatric disorder characterized by several symptoms, including low mood, low self-esteem, and loss of interest or pleasure in normally enjoyable activities (anhedonia). ${ }^{1,2}$ In addition to these impairments, this disorder is also associated with a high suicide risk in affected patients. ${ }^{3}$ The World Health Organization ranks MDD as the fourth leading cause of disability worldwide, and it is estimated that MDD will be the second leading cause of burden of disease in $2030 .{ }^{4}$

Although several studies have been conducted in an attempt to understand the neurobiological basis of MDD, there is not a complete understanding of the mechanisms that account for the depressive symptoms. However, there is a consensus that MDD is a multicausal disorder whose etiology includes genetical, environmental, and neurobiological factors. One of the main environmental factors associated with MDD is stress. Stressful events during life are highly correlated with the onset and progression of MDD. ${ }^{1,2}$

The initial hypothesis regarding the neurobiology of MDD was the monoaminergic hypothesis that correlates a reduction in the synaptic levels of monoamines (noradrenaline, serotonin, and dopamine) with depressive symptoms. However, this hypothesis does not explain the fact that remission of the symptoms only occurs after several weeks of treatment with conventional antidepressants (selective serotonin reuptake inhibitors, tricyclics, and atypicals), whereas the enhancement in monoamine levels is observed immediately after the administration of these agents..$^{1,5,6}$ This lag in the 
symptom resolution of conventional antidepressants has led to the assumption that neuroplastic alterations dependent on signaling pathways and modulation of target genes account for the slow therapeutic effects of antidepressants. ${ }^{7}$ Preclinical and clinical studies have reported that remission of depressive symptoms depends on the increase in brain-derived neurotrophic factor (BDNF), predominantly in the hippocampus and prefrontal cortex, and hippocampal neurogenesis elicited by antidepressant treatments. ${ }^{8,9}$ This is particularly important considering that MDD is associated with atrophy and loss of neurons and glia, leading to decreased size and function of the prefrontal cortex and hippocampus. ${ }^{7,10}$

The glutamatergic system has received particular attention as a target for the development of antidepressant agents with better pharmacological profiles than currently used conventional antidepressants. ${ }^{11}$ The first preclinical study that showed the antidepressant-like effects of $N$-methyl-D-aspartate (NMDA) receptor antagonists (AP7 and MK-801) was published in 1990 by Trullas and Skolnick. ${ }^{12}$ A review by Skolnick ${ }^{13}$ proposed that NMDA receptor antagonists would be antidepressants for the new millennium. This result was bolstered by several preclinical, and clinical studies that have shown that ketamine, an NMDA receptor antagonist, exhibits fast and sustained antidepressant effects. Ketamine has been shown to be effective in patients who were resistant to two or more classes of standard antidepressants. ${ }^{14-16}$ The first clinical study published on ketamine reported that depressive symptoms decreased with ketamine within 240 minutes and were maintained for at least 3 days. ${ }^{14}$ In another study, ketamine was effective within 2 hours of infusion, and $71 \%$ of patients were responsive to treatment within 1 day. ${ }^{16}$ Subsequent studies have confirmed the efficacy of ketamine, but not for all patients. The response ratio in the clinical studies on ketamine is in the range $25 \%-71 \% 24$ hours postinfusion and $8 \%-45 \%$ 1 week postinfusion. ${ }^{17}$ Preclinical studies have also revealed that ketamine acutely or chronically presents antidepressant effects in rodents submitted to animal models of depression based on the exposure of rodents to stress, including maternal deprivation and chronic mild stress. ${ }^{18,19}$

The fast-acting clinical effect of ketamine has led to a greater interest in the molecular mechanism underlying its antidepressant action. The NMDA receptor antagonism on gamma-aminobutyric acid (GABA)-ergic interneurons with the consequent increase on glutamate levels, followed by the activation of $\alpha$-amino-3-hydroxy5-methyl-4-isoxazolepropionic acid (AMPA) receptors and voltage-dependent calcium channels are fundamental steps that lead to an increase in BDNF release in the prefrontal cortex and hippocampus. As a consequence of these events, activation of mammalian target of rapamycin (mTOR) seems to be another fundamental step that accounts for the antidepressant effect of ketamine, since it stimulates the expression of synaptic proteins that are thought to be associated with synaptogenesis and its rapid antidepressant response. ${ }^{20}$ Considering these findings, mTOR activation stands out as a key target for antidepressant responses. Therefore, this review presents the main preclinical and clinical evidence that supports this assumption.

\section{The role of $\mathrm{mTOR}$ in the central nervous system}

mTOR, a 289 kDa evolutionarily conserved serine/threonine protein kinase, may be activated by phosphorylation in response to growth factors (such as BDNF), mitogens, and stress. ${ }^{21,22}$ It has been recognized to have an essential role in the regulation of protein synthesis, energy metabolism, lipid metabolism, cell growth and size, autophagy, and lysosome biogenesis. ${ }^{21-23}$ In the brain, mTOR is also involved in axonal sprouting, axonal regeneration and myelination, ionic and receptor channel expression, dendritic spine growth, and astrocyte migration and proliferation. ${ }^{23,24}$ mTOR-regulated processes in the brain influence neuronal excitability, neuronal survival, synaptic and behavioral plasticity, cognition, feeding, and control of circadian rhythm. ${ }^{23}$

The function of mTOR signaling is mediated via two mTOR complexes: mTORC1 and mTORC2. ${ }^{23}$ mTOR function is influenced by the activities of several receptors, including NMDA, AMPA, tropomyosin receptor kinase B (TrkB), dopaminergic, and metabotropic glutamate receptors (mGluRs). ${ }^{25-27}$ NMDA receptor activation is associated with phosphorylation of intracellular pathways, including protein kinase B (Akt) and protein phosphatases that in turn can activate glycogen synthase kinase-3 (GSK-3). GSK-3 has been reported to suppress mTOR signaling in the hippocampus. ${ }^{28}$ In addition, the inhibition of GSK-3 is required for the antidepressant effects of ketamine. ${ }^{29,30}$

Activated mTOR phosphorylates the $70 \mathrm{kDa}$ ribosomal protein S6 kinase 1 (p70S6K) and also phosphorylates and inactivates the eukaryotic initiation factor 4E-binding protein 1 (4E-BP1) reducing its affinity for the eukaryotic initiation factor 4E (eIF4E), thus releasing eIF4E to facilitate translation initiation. ${ }^{31}$ mTORC1 has been shown to be associated with protein translation. It is formed by a catalytic subunit, mTOR, and Raptor, which is an mTOR-binding protein that also binds to p70S6K and 4E-BP1. Moreover, 
mTORC1 complex activity is stimulated by oxidative stress and inflammation, ${ }^{32}$ and by growth factors through both phosphoinositide 3 kinase (PI3K)-Akt and Ras-extracellular signal-regulated kinase (ERK)-mediated pathways. ${ }^{33}$ In contrast, mTORC2 regulates the development of the cytoskeleton and also controls cell survival. ${ }^{34}$

mTORC1 is sensitive to inhibition by the macrolide antibiotic rapamycin. Conversely, mTORC2 is relatively resistant to rapamycin, and prolonged treatment is required for rapamycin to inhibit the activity of mTORC2. ${ }^{35}$ Rapamycin inhibits the P70S6K and 4E-BP1 phosphorylation, thus reducing transcription and translation of protein mediated by mTOR signaling. It prevents mTOR from further phosphorylation of P70S6K, 4E-BP1, thus indirectly decreasing the proteins involved in transcription and translation of this signaling. A body of evidence has found that rapamycin exhibits anti-proliferation and anti-migration properties in many types of cells. ${ }^{36-38}$

mTOR is also known to exert an important role in the development of the brain. This effect appears to be mediated by BDNF and includes learning and memory formation in the hippocampus and synaptogenesis in the prefrontal cortex..$^{33,39-42}$ The formation of learning and memory mediated by $\mathrm{mTORC} 1$ is promoted via protein synthesis-dependent strengthening of synapses..$^{40}$ The role of mTOR in memory is supported by the fact that hyperactivation of PI3K/Akt/mTOR signaling was shown in postmortem samples of the inferior parietal lobule in patients with Alzheimer's disease (AD) ${ }^{43}$

Excessive mTOR activity is involved in maladaptive memory and learning and with abuse disorders. For example, alcohol administration in mice increased mTOR activation in the nucleus accumbens, a brain area associated with addiction and reward. ${ }^{44}$ On the other hand, decreased mTOR and associated cascades are also involved with learning and memory impairment. In a preclinical study, memory, learning, and social behavior impairment in mice deficient of 4E-BP1 was reported. ${ }^{45,46}$ Changes in nutrition are also associated with altered mTOR activity. In some parts of hypothalamus in which there are higher ambient levels of phosphorylated S6K1 (a marker for mTORC1 activity), this activity decreases during fasting conditions. ${ }^{47}$ For instance, Su et $\mathrm{al}^{42}$ demonstrated that a long-term protein-rich diet restored learning and memory impairment in rats via upregulation of mTOR/ p70S6K signaling.

Since mTOR is involved in memory processes and neuronal plasticity, it stands to reason that several studies have correlated mTOR activity and its associated signaling cascades with the pathophysiology of some nervous system disorders such as Parkinson's disease, AD, Huntington's disease, bipolar disorder, and MDD..$^{33,48,49}$ Thus, targeting the mTOR pathway could be very important in understand the pathophysiology of these diseases and for the development of new therapeutic strategies.

\section{Preclinical evidence supporting the role of $m$ TOR signaling in the neuropathophysiology of MDD}

The activation of several intracellular signaling pathways plays a role in synaptic neurotransmission and plasticity, mechanisms critical to cell survival, and mood regulation. ${ }^{50}$ In the last few years, special attention has been given to the role of mTOR signaling in mood modulation. Several studies have reported decreased brain $\mathrm{mTOR}$ activation in animal models of depression (Figure 1). Regarding these models, one of the most studied is chronic unpredictable stress (CUS), which mimics several behavioral and neurochemical alterations that occur in depressive individuals. In this model, rodents are exposed to long-term, mild, but unpredictable stressors and develop a state of impaired reward salience that mimics the anhedonia observed in many depressive patients. ${ }^{51}$

Mice and rats exposed to CUS exhibit depressive-like behaviors associated with a reduction in phosphorylation levels of mTOR and its downstream signaling components, such as phosphor-p70S6K, in the prefrontal cortex, ${ }^{20,52}$ hippocampus, ${ }^{53}$ and amygdala. ${ }^{54}$ Also, the genetic deletion of $\mathrm{mTOR}$ in mice recapitulates depressive-like behaviors induced by CUS. ${ }^{53}$ The successive administration of corticosterone to mouse cortical neuron cultures, a model that resembles the effects of stress, was also shown to reduce the phosphorylation levels of mTOR. ${ }^{55}$ However, rats submitted to 10-day restraint stress presented an overexpression of mTOR in the hippocampus. ${ }^{56}$ In the model of depression induced by neonatal clomipramine administration, the phosphorylation of p70S6 kinase, one of the mTOR downstream effectors, was significantly decreased in the hippocampus, hypothalamus, and frontal cortex of clomipramine-treated rats, suggesting that reduction in $\mathrm{mTOR}$ activation is associated with depression-related situations. ${ }^{57}$

Regarding the role of mTOR signaling in the behavioral response of antidepressant drugs, an elegant study that has provided insight into the role of this protein in the neuropathophysiology of MDD was published by Li et al. ${ }^{20}$ In this study, a single dose of ketamine was able to activate the mTOR signaling pathway, resulting in increased prefrontal cortex synaptic protein expression within 2 hours and increased dendritic spine density and synaptic activity 


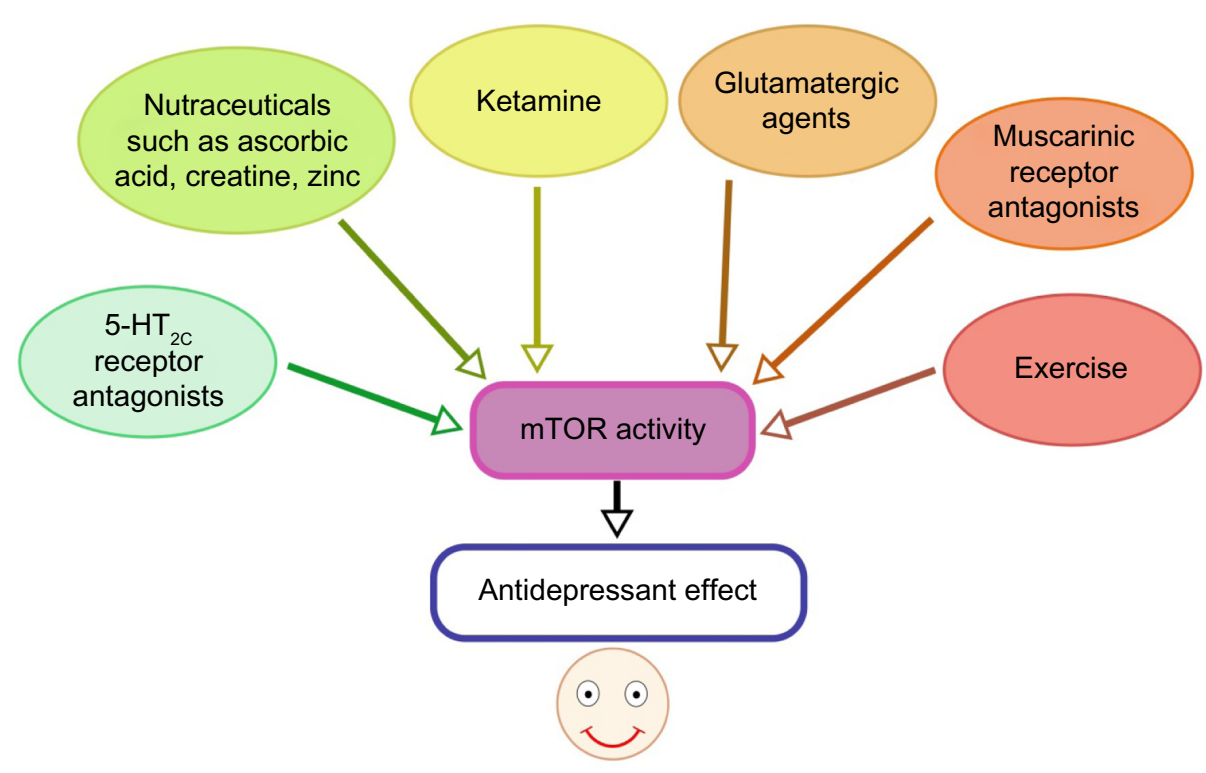

Figure I Potential strategies for the management of MDD targeting mTOR signaling.

Notes: Several studies have suggested that the activation of mTOR may elicit antidepressant effects. Ketamine and some glutamatergic agents, including ascorbic acid, creatine, zinc, and guanosine, as well as mGluR $_{2 / 3}$ antagonist LY34I495, mGlu7 agonist AMN082, the glycine binding site NMDA receptor antagonist 7-CTKA, and NMDAR glycine site functional partial agonist GLYX-13 which present antidepressant properties, are reported to cause mTOR activation. Also, 5-HT $2 C$ receptor antagonists, muscarinic receptor antagonists, and physical exercise may cause mTOR activation.

Abbreviations: MDD, major depressive disorder; mTOR, mammalian target of rapamycin; NMDA, N-methyl-D-aspartate; mGluR, metabotropic glutamate receptor; NMDAR, N-methyl-D-aspartate receptor; 5-HT2C, 5-hydroxytryptamine 2C.

within 24 hours. The synaptic actions of ketamine allow rapid recovery from the insults produced by exposure to repeated stress that cause neuronal atrophy and loss of synaptic connections. Ketamine's effects on behavior and synaptogenesis in the prefrontal cortex seem to be mediated by activation of mTOR signaling, since rapamycin administration was able to prevent ketamine's effects. ${ }^{20,28}$ These results are particularly important in elucidating ketamine's therapeutic mechanism of action and fast-acting antidepressant properties.

It was also demonstrated that the acute administration of ketamine increased BDNF levels and p-mTOR in the hippocampus of rats and produced an antidepressant effect in the forced swimming test (FST). ${ }^{58}$ More recently, a study by Zhou et $\mathrm{al}^{41}$ also showed the antidepressant-like effect of ketamine during the FST associated with an increase in hippocampal and prefrontal cortical mTOR phosphorylation and enhanced BDNF levels. These effects were attenuated or abolished (depending on the dose) by the AMPA receptor antagonist NBQX. On the other hand, the ability of ketamine to increase mTOR phosphorylation and BDNF levels in both brain structures was potentiated by the AMPA receptor agonist CX546. These results indicate that the antidepressant effect of ketamine is associated with upregulation of mTOR and BDNF through AMPA receptor activation in the hippocampus and prefrontal cortex of rats (Figure 2). These conclusions are corroborated by the finding that ketamine treatment stimulates BDNF release in primary cortical

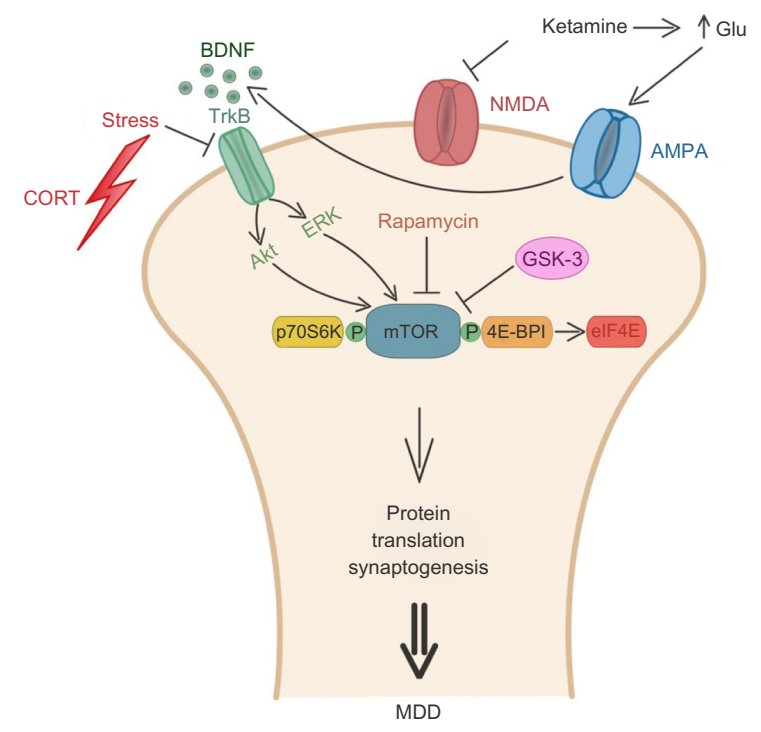

Figure 2 Role of mTOR signaling pathway in the pathophysiology of MDD.

Notes: $m$ TOR function is influenced by the activities of several receptors, including NMDA, AMPA, and TrkB. Exposure to chronic stress or corticosterone can reduce mTOR activation, whereas the activation of GSK-3 is associated with mTOR inhibition. Ketamine and other compounds cause mTOR activation. Particularly, ketamine activates $m$ TOR through the activation of TrkB receptors as a consequence of BDNF release due to AMPA receptor activation. On the other hand, rapamycin inhibits mTOR activity and blocks antidepressant effects of ketamine and other glutamatergic agents. Activated mTORCI phosphorylates p70S6K and also phosphorylates and inactivates $4 \mathrm{E}-\mathrm{BPI}$, reducing its affinity for elF4E. Reduced mTOR activity is generally associated with depressive symptoms.

Abbreviations: AMPA, $\alpha$-amino-3-hydroxy-5-methyl-4-isoxazolepropionic acid; BDNF, brain-derived neurotrophic factor; 4E-BPI, eukaryotic initiation factor 4E-binding protein I; CORT, corticosterone; elF4E, eukaryotic initiation factor 4E; GSK-3, glycogen synthase kinase-3; MDD, major depressive disorder; mTOR, mammalian target of rapamycin; NMDA, $\mathrm{N}$-methyl-D-aspartate; p70S6K, 70-kDa ribosomal protein S6 kinase I; TrkB, tropomyosin receptor kinase B; ERK, Ras-extracellular signal-regulated kinase; Akt, protein kinase $B$. 
neurons, an effect blocked by inhibition of AMPA receptors. ${ }^{59}$ In addition, this study showed that BDNF antibody administered into the prefrontal cortex blocked the antidepressant effect of ketamine in the FST. L-type calcium channel antagonists also abolished the behavioral effects of ketamine in this test, suggesting that the activation of L-type calcium channels that occurs as a consequence of AMPA receptor activation is associated with the antidepressant effect of ketamine. Also, ketamine-mediated blockade of NMDA receptors at rest causes effects compatible with mTOR activation, namely the deactivation of eukaryotic elongation factor 2 (eEF2) kinase, leading to reduced eEF2 phosphorylation and the consequent BDNF translation. ${ }^{60}$

Considering the relationship between GSK-3 and mTOR, ${ }^{29,30}$ it is not surprising that inhibition of GSK-3 is required for the rapid antidepressant-like effect of ketamine. A study by Beurel et $\mathrm{al}^{29}$ showed that treatment of mice with ketamine rapidly increases phosphorylation of both $\alpha$ and $\beta$ isoforms of GSK-3, causing an inhibition of GSK-3 activity and that mice carrying a mutant form of GSK-3 that prevents its phosphorylation are resistant to the antidepressant-like effect of ketamine in learned helplessness.

Another antidepressant strategy in preclinical studies has been the association of subeffective doses of ketamine and other compounds. For example, tramadol enhanced the ketamine-induced antidepressant effects and upregulated the expression of mTOR in the hippocampus and prefrontal cortex of rats. ${ }^{61}$ The effects of ketamine on the FST and on synaptogenesis in the prefrontal cortex were potentiated by the GSK-3 inhibitors lithium and SB 216763. ${ }^{30}$ A study by Chiu et $\mathrm{al}^{62}$ also reported that the combination of subeffective doses of lithium and ketamine caused an antidepressant-like effect in the FST associated with activation of mTOR signaling pathway in prefrontal cortex. Finally, the combined administration of subeffective doses of AMPA and ketamine caused antidepressant effect in the FST and sucrose preference test associated with increases in hippocampal BDNF, synapsin levels, and mTOR phosphorylation in rats. ${ }^{63}$

A number of additional compounds have also been reported to possess antidepressant properties primarily by modulating glutamatergic neurotransmission and may activate mTOR in prefrontal cortex and/or hippocampus (Figure 1). Ascorbic acid, a putative neuromodulator with antioxidant properties that possesses neuroprotective effects against glutamate-induced neurotoxicity, ${ }^{64,65}$ produced antidepressant-like effects in several behavioral tests ${ }^{66-68}$ and was reported to afford beneficial effects against depressive symptoms $^{69}$ and to increase mood in healthy young adults. ${ }^{70}$ A single administration of ascorbic acid increased the phosphorylation p70S6K, a downstream target to mTOR, and the immunocontent of the synaptic protein PSD-95 in the hippocampus of mice. Of note, this effect occurred just 1 hour after its administration. In addition, rapamycin was able to abolish the antidepressant-like effect of ascorbic acid in the tail suspension test (TST). ${ }^{71}$ In a mechanism similar to that of ascorbic acid, mTOR signaling was also implicated in the antidepressant-like effect of guanosine in the TST, ${ }^{72}$ a purine nucleoside that has been shown to modulate glutamatergic neurotransmission..$^{73}$ More recently, it was demonstrated that creatine, an endogenous ergogenic compound that has shown neuroprotective properties against several agents, including NMDA-induced excitotoxic damage,${ }^{74}$ exerts antidepressantlike effect in the TST by a mechanism dependent on NMDA receptor inhibition, ${ }^{75} \mathrm{Akt}$, Nrf2/HO-1, GPx, and mTOR activation, and GSK-3 inhibition. ${ }^{76}$ Creatine administered acutely increased the phosphorylation of p70S6K and Akt, proteins downstream, and upstream to mTOR, respectively, suggesting that $\mathrm{mTOR}$ activation may account for its antidepressantlike effects. This assumption was reinforced by the finding that rapamycin abolished the antidepressant-like effect of creatine in the $\mathrm{TST}^{76}$ More recently, it was shown that the activation of mTOR signaling pathway is also implicated in the antidepressant-like effect of zinc, a metal ion that inhibits NMDA receptors. ${ }^{77}$

Besides the putative ability of the glutamatergic modulators ascorbic acid, guanosine, creatine, and zinc to produce antidepressant effects by modulating mTOR signaling, erythropoietin, scopolamine, and other glutamatergic agents seem to act via this mechanism.

Erythropoietin and the muscarinic receptor antagonist scopolamine were reported to produce an antidepressantlike effects in the FST, and rapamycin was able to abolish these effects. ${ }^{78,79}$ Erythropoietin also promoted adult hippocampal neurogenesis, an effect exhibited by effective antidepressants. ${ }^{78}$ Besides mTOR signaling, the antidepressant effects of scopolamine are associated with increased glutamate transmission, and synaptogenesis, similar to ketamine. ${ }^{79}$

A single dose of the mGluR $_{2 / 3}$ antagonist LY341495 rapidly (1 hour) activated the mTOR pathway (mTOR, p70S6K, 4E-BP1) and subsequently (24 hours later) increased levels of synaptic proteins (PSD-95, GluR1, and synapsin I) in a manner similar to ketamine. Reinforcing the critical role of mTOR for the antidepressant-like effect of LY341495, its anti-immobility effect in the rat FST was abolished by rapamycin. ${ }^{80}$ However, a previous study showed that rapamycin blocked the sustained, but not the acute, antidepressantlike effects of mGlu2/3 receptor antagonists MGS0039 and 
LY341495, suggesting that the activation of mTOR signaling may contribute to the sustained antidepressant-like effects of $\mathrm{mGlu} 2 / 3$ receptor antagonists. ${ }^{81}$

A recent study showed that the mGlu7 agonist AMN082 administered 1 hour before the FST increased the levels of p-mTOR and p-p70S6K, and rapamycin reversed its antidepressant-like effect in the FST in rats. This study showed that synapsin I and GluR1 levels were increased in the prefrontal cortex, further indication that AMN082 acts by activating mTOR signaling. ${ }^{82}$

Reinforcing the notion that mTOR is implicated in the mechanism underlying the fast-acting antidepressants, a single administration of the glycine binding site NMDA receptor antagonist 7-CTKA produced rapid antidepressant-like effects associated with increased p-GSK3 $\beta$ (causing inhibition of this enzyme), enhanced mTOR function, and increased postsynaptic protein levels in the medial prefrontal cortex of rats. ${ }^{52}$ It was also recently shown that GLYX-13, an NMDA receptor (NMDAR) glycine site functional partial agonist, was able to acutely reverse chronic mild stress-induced depressive-like behavior and reduction in Akt and mTOR phosphorylation in the hippocampus of mice. In addition, rapamycin blocked the antidepressant-like effect of GLYX-13. ${ }^{83}$

\section{Clinical evidence supporting the role of $\mathrm{mTOR}$ signaling in the neuropathophysiology of MDD}

A study by Jernigan et $\mathrm{al}^{84}$ investigated the expression of mTOR and its downstream signaling targets: p70S6K, eIF4E, eIF4E phosphorylated at serine 209 (p-eIF4E-Ser209), eIF4B, and eIF4B phosphorylated at serine 504 (p-eIF4B-Ser504) in the prefrontal cortex of 12 depressed individuals and 12 psychiatrically healthy controls. This study showed a reduction in mTOR, p70S6K, eIF4B, and p-eIF4B protein expression in MDD subjects when compared with controls, pointing to a deficit in mTOR-dependent signaling leading to impairment in its downstream targets that control translation of synaptic proteins. A similar result regarding mTOR was shown in blood samples taken from bipolar patients during depressive episodes; mTOR mRNA and Akt mRNA expression was reduced in comparison with healthy controls, alterations that correspond to that observed in brain samples. ${ }^{85}$ Additionally, the treatment of three depressive patients with a subanesthetic dose of ketamine afforded a rapid amelioration of depression symptoms associated with acute increases in the expression of plasma mTOR and GSK-3, as well as eEF2 phosphorylation. ${ }^{86}$

Of note, levels of the NR2A and NR2B subunits of NMDA receptors and the postsynaptic protein PSD95, a target downstream to mTOR, were found to be reduced in postmortem samples of prefrontal cortex Brodmann's area 10 (BA10) in depressed individuals when compared with psychiatrically healthy controls. ${ }^{87}$ However, increased levels of NR2A and PSD-95 were reported in amygdala samples of depressed individuals. ${ }^{88}$ The alterations found in the prefrontal cortex BA10 region may be related to the depressive symptoms, since BA10 is not only associated with executive functions such as planning and integrative information processing, but also in reward and reinforcement processing. ${ }^{89,90}$ Therefore, this area may be involved in mood regulation, considering that depressive patients may exhibit impaired reward responsiveness, characterizing a reduced hedonic behavior. ${ }^{91}$

Postmortem studies have also showed increased expression of mitogen-activated protein kinase (MAPK) phosphatase-1 (MKP-1) in the dentate gyrus and CA1 region of the hippocampus of depressive subjects when compared with matched controls. ${ }^{92}$ This study also demonstrated significant downregulation of mitogen-activated protein kinase (MAPK) kinase 2 (MEK2) in the CA1 region of subjects with depression and a decrease in ERK2, a MAP kinase directly regulated by MKP-1, in the dentate gyrus. This corresponds with a previous postmortem study that showed reduced activation and expression of ERK1/2 MAP kinase in the postmortem prefrontal cortex and hippocampus of depressed suicide subjects. ${ }^{93}$ Considering that MKP-1 is a key negative regulator of the MAPK cascade ${ }^{94}$ that is also upstream to mTOR, it is possible that it can impair mTOR signaling in the prefrontal cortex and hippocampus of depressive individuals.

Altogether, the clinical studies point to a possible association between marked deficits in synaptic proteins and dysregulation of mTOR signaling, mainly in the prefrontal cortex of depressive individuals. The role of mTOR signaling in other brain regions, particularly the hippocampus and amygdala, in the neuropathophysiology of depression remains to be established.

\section{Limitations associated with therapeutic targeting $\mathbf{m T O R}$}

Several studies indicate that inhibition of mTORC1 can be beneficial for some pathological conditions such as epilepsy, cognitive impairment, cancer therapy and as an immunosuppressant to prevent graft rejection in transplant recipients, whereas stimulation of $\mathrm{mTORC} 1$ can be beneficial for depression or axonal growth and regeneration. ${ }^{23}$ The broad therapeutic roles of targeting mTOR are a result of the essential role mTOR signaling plays in several biological 
functions, as previously mentioned in this review. Since depressive symptoms are often present in several pathological conditions associated with brain mTOR stimulation (such as epilepsy, cancer, and AD) targeting mTOR for the treatment of depression has some limitations. It should be noted that a significant percentage of depressive patients would likely not benefit from pharmacological strategies targeting brain mTOR activation. We should also state that glutamatergic agents that activate mTOR, such as ketamine, may not be safe for chronic treatment. Regarding these limitations, modulators of mTOR devoid of psychomimetic effects are needed. However, even with safer agents, the effects of long-term modulation of mTOR have yet to be investigated.

It is also important to note that there are some controversial effects regarding the possible role of mTOR in depression. Chronic treatment with rapamycin for 17 weeks in WAG/Rij (Wistar Albino Glaxo/Rij) rats, a genetic model of absence epilepsy, epileptogenesis, and mild-depression comorbidity, caused antiepileptogenic effects accompanied by prodepressant effects, but subchronic treatment (7 days) with rapamycin had no effect on forced swim behavior in healthy Wistar rats and caused antidepressant-like effects in the WAG/Rij rats. ${ }^{95}$ Also, the antidepressant-like effects of subchronic administration of rapamycin were shown in two predictive models of antidepressant activity, the FST and TST in mice and rats, although the authors proposed that this effect may be dependent on other intracellular interactions besides mTOR inhibition. ${ }^{96}$ Interestingly, sleep deprivation, a nonpharmacological antidepressant treatment that rapidly alleviates depressive symptoms in $\sim 60 \%$ of depressed patients, ${ }^{97}$ was reported to reduce levels of total and phosphorylated mTOR in the mouse hippocampus. ${ }^{98}$ These results suggest that, at least under some conditions, mTOR inhibition may be associated with antidepressant responses. These data indicate that the role of mTOR signaling in mood regulation is complex and requires further studies to investigate the mechanisms underlying these effects.

A recent in vitro study showed that the antidepressants escitalopram, paroxetine, and tranylcypromine, similar to ketamine, significantly increased levels of p-mTOR and its downstream regulators (p-4E-BP1 and p-p70S6K), and increased hippocampal dendritic outgrowth and synaptic proteins levels, whereas fluoxetine, sertraline, and imipramine caused no effect on these parameters in rat hippocampal cultures. ${ }^{99}$ This result is somewhat in agreement with the finding that imipramine or fluoxetine (as well as electroconvulsive treatment) did not affect mTOR signaling in the prefrontal cortex of rats. ${ }^{20}$ These in vitro results suggest that some monoaminergic antidepressants may promote dendritic outgrowth and increase synaptic protein levels through mTOR signaling. However, it remains to be determined whether these antidepressants are able to elicit these effects in vivo. It is possible that these antidepressants do not reach concentrations in vivo that are sufficient to activate mTOR signaling. ${ }^{99}$

Treatment with 5-hydroxytryptamine $2 \mathrm{C}\left(5-\mathrm{HT}_{2 \mathrm{C}}\right)$ receptor antagonists was also reported to induce fast-onset antidepressant effects associated with the activation of $\mathrm{mTOR}$ and eEF2 in the prefrontal cortex of mice. Of note, subchronic treatment with selective serotonin reuptake inhibitors, which did not induce antidepressant behavioral effects, also activated mTOR and eEF2, indicating that these effects are not sufficient for antidepressant onset. ${ }^{100}$

Finally, a transient activation of mTOR was shown in the hippocampus of rats subjected to forced treadmill exercise ( 1 day of exercise, but not 2 or 8 weeks of exercise). ${ }^{101}$ Considering that exercise is associated with antidepressant effects, ${ }^{102}$ this finding deserves further studies to understand the role of mTOR signaling in antidepressant responses.

The exact mechanism by mTOR signaling pathway may modulate mood is unknown. The decreased mTOR signaling may impair synaptic plasticity, cellular resilience, and energy production ${ }^{23}$ that may contribute to the development of MDD.

\section{Conclusion}

In this review, we present basic research data combined with clinical studies indicating that mTOR signaling plays a crucial role in the neuropathophysiology of depression (Figure 2). Overall, it seems that depression is associated with a reduced activity of this enzyme, whereas antidepressant treatments, mainly glutamatergic agents such as ketamine, have been shown to cause an activation of this protein through its phosphorylation, especially in the prefrontal cortex and hippocampus. It remains to be established whether antidepressant agents that cause an activation of this enzyme are able to induce a fast-onset therapeutic response. A better understanding on the role of mTOR signaling in different brain regions and its upstream and downstream targets may provide novel insights into the development of therapeutic approaches for the management of MDD.

\section{Acknowledgments}

The Center for Translational Psychiatry (USA) is funded by the Department of Psychiatry and Behavioral Sciences, The University of Texas Medical School at Houston. Laboratory of Neurosciences (Brazil) is one of the centers of 
the National Institute for Molecular Medicine (INCT-MM). Laboratory of Neurobiology of Depression and Laboratory of Neurosciences (Brazil) are members of the Center of Excellence in Applied Neurosciences of Santa Catarina (NENASC). Work in the authors' laboratories has been funded by grants from CNPq, CAPES, FAPESC, Instituto Cérebro e Mente, and UNESC, Brazil. JQ and ALSR are CNPq Research Fellows.

\section{Disclosure}

The authors report no conflicts of interest in this work.

\section{References}

1. Wong ML, Licinio J. Research and treatment approaches to depression. Nat Rev Neurosci. 2001;2(5):343-351.

2. Nestler EJ, Barrot M, DiLeone RJ, Eisch AJ, Gold SJ, Monteggia LM. Neurobiology of depression. Neuron. 2002;34(1):13-25.

3. Raue PJ, Ghesquiere AR, Bruce ML. Suicide risk in primary care: identification and management in older adults. Curr Psychiatry Rep. 2014;16(9):466

4. Mathers CD, Loncar D. Projections of global mortality and burden of disease from 2002 to 2030. PLoS Med. 2006;3(11):e442.

5. Wong ML, Licinio J. From monoamines to genomic targets: a paradigm shift for drug discovery in depression. Nat Rev Drug Discov. 2004; 3(2):136-151.

6. Krishnan V, Nestler EJ. The molecular neurobiology of depression. Nature. 2008;455:894-902.

7. Duman RS, Voleti B. Signaling pathways underlying the pathophysiology and treatment of depression: novel mechanisms for rapid-acting agents. Trends Neurosci. 2012;35(1):47-56.

8. Duman RS, Monteggia LM. A neurotrophic model for stress-related mood disorders. Biol Psychiatry. 2006;59(12):1116-1127.

9. Schmidt HD, Duman RS. The role of neurotrophic factors in adult hippocampal neurogenesis, antidepressant treatments and animal models of depressive-like behavior. Behav Pharmacol. 2007;18(5-6):391-418.

10. Sheline Y, Gado MH, Kraemer HC. Untreated depression and hippocampal volume loss. Am J Psychiatry, 2003;160(8): $1516-1518$

11. Sanacora G, Treccani G, Popoli M. Towards a glutamate hypothesis of depression: an emerging frontier of neuropsychopharmacology for mood disorders. Neuropharmacology. 2012;62(1):63-77.

12. Trullas R, Skolnick P. Functional antagonists at the NMDA receptor complex exhibit antidepressant actions. Eur J Pharmacol. 1990; 185(1):1-10.

13. Skolnick P. Antidepressants for the new millennium. Eur J Pharmacol. 1999;375(1-3):31-40.

14. Berman RM, Cappiello A, Anand A, et al. Antidepressant effects of ketamine in depressed patients. Biol Psychiatry. 2000;47(4):351-354.

15. Fond G, Loundou A, Rabu C, et al. Ketamine administration in depressive disorders: a systematic review and meta-analysis. Psychopharmacology. 2014;231:3663-3676.

16. Zarate CJ, Singh JB, Carlson PJ, et al. A randomized trial of an $N$-methylD-aspartate antagonist in treatment-resistant major depression. Arch Gen Psychiatry. 2006;63:856-864.

17. Iadarola ND, Niciu MJ, Richards EM, et al. Ketamine and other $N$-methylD-aspartate receptor antagonists in the treatment of depression: a perspective review. Ther Adv Chronic Dis. 2015;6(3):97-114.

18. Garcia LS, Comim CM, Valvassori SS, et al. Ketamine treatment reverses behavioral and physiological alterations induced by chronic mild stress in rats. Prog Neuropsychopharmacol Biol Psychiatry. 2009; 33(3):450-455.
19. Réus GZ, Abelaira HM, dos Santos MA, et al. Ketamine and imipramine in the nucleus accumbens regulate histone deacetylation induced by maternal deprivation and are critical for associated behaviors. Behav Brain Res. 2013;256:451-456.

20. Li N, Lee BY, Liu RJ, et al. mTOR-dependent synapse formation underlies the rapid antidepressant effects of NMDA antagonists. Science. 2010;329:959-964.

21. Dazert E, Hall MN. mTOR signaling in disease. Curr Opin Cell Biol. 2011;23:744-755.

22. Laplante M, Sabatini DM. mTOR signaling in growth control and disease. Cell. 2012;149:274-293.

23. Bockaert J, Marin P. mTOR in brain physiology and pathologies. Physiol Rev. 2015; 95(4): 1157-1187.

24. Li CY, Li X, Liu SF, Qu WS, Wang W, Tian DS. Inhibition of mTOR pathway restrains astrocyte proliferation, migration and production of inflammatory mediators after oxygen-glucose deprivation and reoxygenation. Neurochem Int. 2015;83-84:9-18.

25. Dadalko OI, Siuta M, Poe A, et al. mTORC2/Rictor signaling disrupts dopamine-dependent behaviors via defects in striatal dopamine neurotransmission. J Neurosci. 2015;35:8843-8854.

26. Hsu WL, Chung HW, Wu CY, et al. Glutamate stimulates local protein synthesis in the axons of rat cortical neurons by activating alpha-amino-3-hydroxy-5-methyl-4-isoxazolepropionic acid (AMPA) receptors and metabotropic glutamate receptors. J Biol Chem. 2015;290:20748-20760.

27. Koskimäki J, Matsui N, Umemori J, Rantamäki T, Castrén E. Nimodipine activates TrkB neurotrophin receptors and induces neuroplastic and neuroprotective signaling events in the mouse hippocampus and prefrontal cortex. Cell Mol Neurobiol. 2015;35(2): 189-196.

28. Dwyer JM, Duman RS. Activation of mammalian target of rapamycin and synaptogenesis: role in the actions of rapid-acting antidepressants. Biol Psychiatry. 2013;73(12):1189-1198.

29. Beurel E, Song L, Jope RS. Inhibition of glycogen synthase kinase-3 is necessary for the rapid antidepressant effect of ketamine in mice. Mol Psychiatry. 2011;16(11):1068-1070.

30. Liu RJ, Fuchikami M, Dwyer JM, Lepack AE, Duman RS, Aghajanian GK. GSK-3 inhibition potentiates the synaptogenic and antidepressant-like effects of subthreshold doses of ketamine. Neuropsychopharmacology. 2013;38(11):2268-2277.

31. Fingar DC, Richardson CJ, Tee AR, Cheatham L, Tsou C, Blenis J. mTOR controls cell cycle progression through its cell growth effectors S6K1 and 4E-BP1/eukaryotic translation initiation factor 4E. Mol Cell Biol. 2004;24:200-216.

32. Lu CL, Qin L, Liu HC, Candas D, Fan M, Li JJ. Tumor cells switch to mitochondrial oxidative phosphorylation under radiation via mTORmediated hexokinase II inhibition-a Warburg-reversing effect. PLoS One. 2015;10:e121046.

33. Garelick MG, Kennedy BK. TOR on the brain. Exp Gerontol. 2011; 46(2-3):155-163.

34. Chong ZZ, Shang YC, Zhang L, Wang S, Maiese K. Mammalian target of rapamycin: hitting the bull's-eye for neurological disorders. Oxid Med Cell Longev. 2010;3(6):374-391.

35. Mohindra NA, Platanias LC. Catalytic mammalian target of rapamycin inhibitors as antineoplastic agents. Leuk Lymphoma. 2015:1-6.

36. Jin Y, Qu S, Tesikova M, et al. Molecular circuit involving KLK4 integrates androgen and mTOR signaling in prostate cancer. Proc Natl Acad Sci U S A. 2013;110(28):E2572-E2581.

37. Liu D, Xing J, Trink B, Xing M. BRAF mutation-selective inhibition of thyroid cancer cells by the novel MEK inhibitor RDEA119 and genetic-potentiated synergism with the mTOR inhibitor temsirolimus. Int J Cancer. 2010;127(12):2965-2973.

38. Molhoek KR, Brautigan DL, Slingluff CL Jr. Synergistic inhibition of human melanoma proliferation by combination treatment with B-Raf inhibitor BAY43-9006 and mTOR inhibitor rapamycin. J Transl Med. 2005;3:39. 
39. Swiech L, Perycz M, Malik A, Jaworski J. Role of mTOR in physiology and pathology of the nervous system. Biochim Biophys Acta. 2008;1784:116-132.

40. Hoeffer CA, Klann E. mTOR signaling: at the crossroads of plasticity, memory and disease. Trends Neurosci. 2010;33:67-75.

41. Zhou W, Wang N, Yang C, Li XM, Zhou ZQ, Yang JJ. Ketamine-induced antidepressant effects are associated with AMPA receptors-mediated upregulation of $\mathrm{mTOR}$ and BDNF in rat hippocampus and prefrontal cortex. Eur Psychiatry. 2014;29(7):419-423.

42. Su ZW, Liao JY, Zhang H, et al. Postnatal high-protein diet improves learning and memory in premature rats via activation of mTOR signaling. Brain Res. 2015;1611:1-7.

43. Tramutola A, Triplett JC, Di Domenico F, et al. Alteration of mTOR signaling occurs early in the progression of Alzheimer disease (AD) analysis of brain from subjects with pre-clinical AD, amnestic mild cognitive impairment and late-stage AD. J Neurochem. 2015;133: 739-749.

44. Neasta J, Barak S, Hamida SB, Ron D. mTOR complex 1: a key player in neuroadaptations induced by drugs of abuse. J Neurochem. 201 130(2):172-184.

45. Banko JL, Poulin F, Hou L, DeMaria CT, Sonenberg N, Klann E. The translation repressor 4E-BP2 is critical for eIF4F complex formation, synaptic plasticity, and memory in the hippocampus. $J$ Neurosci. 2005;25:9581-9590.

46. Banko JL, Merhav M, Stern E, Sonenberg N, Rosenblum K, Klann E Behavioral alterations in mice lacking the translation repressor 4E-BP2. Neurobiol Learn Mem. 2007;87:248-256.

47. Cota D, Proulx K, Smith KA, et al. Hypothalamic mTOR signaling regulates food intake. Science. 2006;312(5775):927-930.

48. Abelaira HM, Réus GZ, Neotti MV, Quevedo J. The role of mTOR in depression and antidepressant responses. Life Sci. 2014;101(1-2): $10-14$.

49. Maiese K. Driving neural regeneration through the mammalian target of rapamycin. Neural Regen Res. 2014;9(15):1413-1417.

50. Pilar-Cúellar F, Vidal R, Díaz A, et al. Signaling pathways involved in antidepressant-induced cell proliferation and synaptic plasticity. Curr Pharm Des. 2014;20(23):3776-3794.

51. Hill MN, Hellemans KG, Verma P, Gorzalka BB, Weinberg J. Neurobiology of chronic mild stress: parallels to major depression. Neurosci Biobehav Rev. 2012;36(9):2085-2117.

52. Zhu W, Wang S, Liu M, et al. Glycine site $N$-methyl-D-aspartate receptor antagonist 7-CTKA produces rapid antidepressant-like effects in male rats. J Psychiatry Neurosci. 2013;38(5):306-316.

53. Zhong P, Wang W, Pan B, et al. Monoacylglycerol lipase inhibition blocks chronic stress-induced depressive-like behaviors via activation of mTOR signaling. Neuropsychopharmacology. 2014;39(7): 1763-1776.

54. Chandran A, Iyo AH, Jernigan CS, Legutko B, Austin MC, Karolewicz B. Reduced phosphorylation of the mTOR signaling pathway components in the amygdala of rats exposed to chronic stress. Prog Neuropsychopharmacol Biol Psychiatry. 2013;40:240-245.

55. Howell KR, Kutiyanawalla A, Pillai A. Long-term continuous corticosterone treatment decreases VEGF receptor-2 expression in frontal cortex. PLoS One. 2011;6(5):e20198.

56. Orlovsky MA, Dosenko VE, Spiga F, Skibo GG, Lightman SL. Hippocampus remodeling by chronic stress accompanied by GR, proteasome and caspase-3 overexpression. Brain Res. 2014;1593: 83-94.

57. Feng P, Huang C. Phospholipase D-mTOR signaling is compromised in a rat model of depression. J Psychiatr Res. 2013;47(5):579-585.

58. Yang C, Hu YM, Zhou ZQ, Zhang GF, Yang JJ. Acute administration of ketamine in rats increases hippocampal BDNF and mTOR levels during forced swimming test. Ups J Med Sci. 2013;118(1):3-8.

59. Lepack AE, Fuchikami M, Dwyer JM, Banasr M, Duman RS. BDNF release is required for the behavioral actions of ketamine. Int $J$ Neuropsychopharmacol. 2014;18(1):1-6.
60. Autry A, Adachi M, Nosyreva E, et al. NMDA receptor blockade at rest triggers rapid behavioural antidepressant responses. Nature. 2011;475: 91-95.

61. Yang C, Li WY, Yu HY, et al. Tramadol pretreatment enhances ketamine-induced antidepressant effects and increases mammalian target of rapamycin in rat hippocampus and prefrontal cortex. J Biomed Biotechnol. 2012;2012:175619.

62. Chiu CT, Scheuing L, Liu G, et al. The mood stabilizer lithium potentiates the antidepressant-like effects and ameliorates oxidative stress induced by acute ketamine in a mouse model of stress. Int $J$ Neuropsychopharmacol. 2014;18(6).

63. Akinfiresoye L, Tizabi Y. Antidepressant effects of AMPA and ketamine combination: role of hippocampal BDNF, synapsin, and mTOR. Psychopharmacology. 2013;230(2):291-298.

64. Rice ME. Ascorbate regulation and its neuroprotective role in the brain. Trends Neurosci. 2000;23(5):209-216.

65. Ballaz S, Morales I, Rodríguez M, Obeso JA. Ascorbate prevents cell death from prolonged exposure to glutamate in an in vitro model of human dopaminergic neurons. J Neurosci Res. 2013;91(12): 1609-1617.

66. Binfaré RW, Rosa AO, Lobato KR, Santos AR, Rodrigues AL. Ascorbic acid administration produces an antidepressant-like effect: evidence for the involvement of monoaminergic neurotransmission. Prog Neuropsychopharmacol Biol Psychiatry. 2009;33(3):530-540.

67. Moretti M, Colla A, de Oliveira Balen G, et al. Ascorbic acid treatment, similarly to fluoxetine, reverses depressive-like behavior and brain oxidative damage induced by chronic unpredictable stress. J Psychiatr Res. 2012;46(3):331-340.

68. Moretti M, Budni J, Freitas AE, et al. TNF- $\alpha$-induced depressivelike phenotype and p38(MAPK) activation are abolished by ascorbic acid treatment. Eur Neuropsychopharmacol. 2015;25(6): 902-912.

69. Amr M, El-Mogy A, Shams T, Vieira K, Lakhan SE. Efficacy of vita$\min \mathrm{C}$ as an adjunct to fluoxetine therapy in pediatric major depressive disorder: a randomized, double-blind, placebo-controlled pilot study. Nutr J. 2013;12:31.

70. Brody S. High-dose ascorbic acid increases intercourse frequency and improves mood: a randomized controlled clinical trial. Biol Psychiatry. 2002;52(4):371-374.

71. Moretti M, Budni J, Freitas AE, Rosa PB, Rodrigues AL. Antidepressantlike effect of ascorbic acid is associated with the modulation of mammalian target of rapamycin pathway. J Psychiatr Res. 2014;48: 16-24.

72. Bettio LE, Cunha MP, Budni J, et al. Guanosine produces an antidepressant-like effect through the modulation of NMDA receptors, nitric oxide-cGMP and PI3K/mTOR pathways. Behav Brain Res. 2012; 234(2):137-148

73. Schmidt AP, Lara DR, Souza DO. Proposal of a guanine-based purinergic system in the mammalian central nervous system. Pharmacol Ther. 2007;116(3):401-416.

74. Beal MF. Neuroprotective effects of creatine. Amino Acids. 2011;40(5): 1305-1313.

75. Cunha MP, Pazini FL, Ludka FK, et al. The modulation of NMDA receptors and L-arginine/nitric oxide pathway is implicated in the antiimmobility effect of creatine in the tail suspension test. Amino Acids. 2015;47(4):795-811.

76. Cunha MP, Budni J, Ludka FK, et al. Involvement of PI3K/Akt signaling pathway and its downstream intracellular targets in the antidepressantlike effect of creatine. Mol Neurobiol. In press 2015.

77. Szewczyk B, Pochwat B, Rafało A, Palucha-Poniewiera A, Domin H, Nowak G. Activation of mTOR dependent signaling pathway is a necessary mechanism of antidepressant-like activity of zinc. Neuropharmacology. 2015;99:517-526.

78. Osborn M, Rustom N, Clarke M, et al. Antidepressant-like effects of erythropoietin: a focus on behavioural and hippocampal processes. PLoS One. 2013;8(9):e72813. 
79. Voleti B, Navarria A, Liu RJ, et al. Scopolamine rapidly increases mammalian target of rapamycin complex 1 signaling, synaptogenesis, and antidepressant behavioral responses. Biol Psychiatry. 2013;74(10):742-749.

80. Dwyer JM, Lepack AE, Duman RS. mTOR activation is required for the antidepressant effects of $\mathrm{mGluR}_{2 / 3}$ blockade. Int $J$ Neuropsychopharmacol. 2012;15(4):429-434.

81. Koike H, Iijima M, Chaki S. Involvement of the mammalian target of rapamycin signaling in the antidepressant-like effect of group II metabotropic glutamate receptor antagonists. Neuropharmacology. 2011;61(8):1419-1423.

82. Pałucha-Poniewiera A, Szewczyk B, Pilc A. Activation of the mTOR signaling pathway in the antidepressant-like activity of the mGlu5 antagonist MTEP and the mGlu7 agonist AMN082 in the FST in rats. Neuropharmacology. 2014;82:59-68.

83. Lu Y, Wang C, Xue Z, et al. PI3K/AKT/mTOR signaling-mediated neuropeptide VGF in the hippocampus of mice is involved in the rapid onset antidepressant-like effects of GLYX-13. Int J Neuropsychopharmacol. 2014;18(5).

84. Jernigan CS, Goswami DB, Austin MC, et al. The mTOR signaling pathway in the prefrontal cortex is compromised in major depressive disorder. Prog Neuropsychopharmacol Biol Psychiatry. 2011; 35(7):1774-1779

85. Machado-Vieira R, Zanetti MV, Teixeira AL, et al. Decreased Akt1/ Mtor pathway mRNA expression in short-term bipolar disorder. Eur Neuropsychopharmacol. 2015;25(4):468-473.

86. Yang C, Zhou ZQ, Gao ZQ, Shi JY, Yang JJ. Acute increases in plasma mammalian target of rapamycin, glycogen synthase kinase-3 $\beta$, and eukaryotic elongation factor 2 phosphorylation after ketamine treatment in three depressed patients. Biol Psychiatry. 2013;73(12):e35-e36.

87. Feyissa AM, Chandran A, Stockmeier CA, Karolewicz B. Reduced levels of NR2A and NR2B subunits of NMDA receptor and PSD-95 in the prefrontal cortex in major depression. Prog Neuropsychopharmacol Biol Psychiatry. 2009;33(1):70-75.

88. Karolewicz B, Szebeni K, Gilmore T, Maciag D, Stockmeier CA, Ordway GA. Elevated levels of NR2A and PSD-95 in the lateral amygdala in depression. Int J Neuropsychopharmacol. 2009; 12(2):143-153.

89. Rogers RD, Owen AM, Middleton HC, et al. Choosing between small, likely rewards and large, unlikely rewards activates inferior and orbital prefrontal cortex. J Neurosci. 1999;19(20):9029-9038.
90. Goswami DB, Jernigan CS, Chandran A, et al. Gene expression analysis of novel genes in the prefrontal cortex of major depressive disorder subjects. Prog Neuropsychopharmacol Biol Psychiatry. 2013;43: 126-133.

91. Pizzagalli DA, Iosifescu D, Hallett LA, Ratner KG, Fava M. Reduced hedonic capacity in major depressive disorder: evidence from a probabilistic reward task. J Psychiatr Res. 2008;43(1):76-87.

92. Duric V, Banasr M, Licznerski P, et al. A negative regulator of MAP kinase causes depressive behavior. Nat Med. 2010;16(11): $1328-1332$.

93. Dwivedi Y, Rizavi HS, Roberts RC, Conley RC, Tamminga CA, Pandey GN. Reduced activation and expression of ERK1/2 MAP kinase in the post-mortem brain of depressed suicide subjects. J Neurochem. 2001;77(3):916-928.

94. Jeffrey KL, Camps M, Rommel C, Mackay CR. Targeting dualspecificity phosphatases: manipulating MAP kinase signalling and immune responses. Nat Rev Drug Discov. 2007;6(5):391-403.

95. Russo E, Citraro R, Donato G, et al. mTOR inhibition modulates epileptogenesis, seizures and depressive behavior in a genetic rat model of absence epilepsy. Neuropharmacology. 2013;69:25-36.

96. Cleary C, Linde JA, Hiscock KM, et al. Antidepressive-like effects of rapamycin in animal models: implications for mTOR inhibition as a new target for treatment of affective disorders. Brain Res Bull. 2008;76:469-473.

97. Hemmeter UM, Hemmeter-Spernal J, Krieg JC. Sleep deprivation in depression. Expert Rev Neurother. 2010;10(7):1101-1115.

98. Vecsey CG, Peixoto L, Choi JH, et al. Genomic analysis of sleep deprivation reveals translational regulation in the hippocampus. Physiol Genomics. 2012;44(20):981-991.

99. Park SW, Lee JG, Seo MK, et al. Differential effects of antidepressant drugs on mTOR signalling in rat hippocampal neurons. Int J Neuropsychopharmacol. 2014;17(11):1831-1846.

100. Opal MD, Klenotich SC, Morais M, et al. Serotonin 2C receptor antagonists induce fast-onset antidepressant effects. Mol Psychiatry. 2014;19(10):1106-1114.

101. Elfving B1, Christensen T, Ratner C, Wienecke J, Klein AB. Transient activation of mTOR following forced treadmill exercise in rats. Synapse. 2013;67(9):620-625.

102. Mura G, Moro MF, Patten SB, Carta MG. Exercise as an add-on strategy for the treatment of major depressive disorder: a systematic review. CNS Spectr. 2014;19(6):496-508.
Journal of Receptor, Ligand and Channel Research

\section{Publish your work in this journal}

The Journal of Receptor, Ligand and Channel Research is an international, peer reviewed, open access, online journal. The journal welcomes laboratory and clinical findings in the fields of biological receptors, ligands, channel and signal transduction research including: receptors and signaling; ligands; transporters, pores and channels; binding and activation; receptor

\section{Dovepress}

regulation; role of receptors in diseases and their treatment; molecular basis of membrane structure and functions; molecular models of membranes. The manuscript management system is completely online and includes a very quick and fair peer-review system. Visit http://www.dovepress.com/ testimonials.php to read real quotes from published authors. 